\title{
Case Report \\ Kerion Celsi in a Nepalese Boy: An Underdiagnosed Cause of Scalp Swelling
}

\author{
Ranjana Parajuli $\mathbb{D}^{1}{ }^{1}$ Ashish Lal Shrestha, ${ }^{2}$ Niranjan Nayak, ${ }^{3}$ Shishir Gokhale, ${ }^{3}$ \\ Kundan Gautam, ${ }^{1}$ and Shishir Subedi ${ }^{1}$ \\ ${ }^{1}$ Department of Microbiology, Grande International Hospital, Dhapasi, Tokha Road, Kathmandu, Nepal \\ ${ }^{2}$ Department of Pediatric Surgery, Grande International Hospital, Dhapasi, Tokha Road, Kathmandu, Nepal \\ ${ }^{3}$ Department of Medical Microbiology, Manipal College of Medical Sciences, Pokhara, Nepal
}

Correspondence should be addressed to Ranjana Parajuli; drranju26@yahoo.com

Received 16 February 2021; Revised 18 May 2021; Accepted 25 May 2021; Published 24 June 2021

Academic Editor: Gloria Taliani

Copyright (C) 2021 Ranjana Parajuli et al. This is an open access article distributed under the Creative Commons Attribution License, which permits unrestricted use, distribution, and reproduction in any medium, provided the original work is properly cited.

\begin{abstract}
Tinea capitis (TC) is an infection of the scalp and hair caused by a dermatophyte fungus. Typically caused by the zoophilic and geophilic species of Microsporum and Trichophyton, it remains the commonest cutaneous fungal infection in children. A 9-yearold Nepalese boy was evaluated in outpatients for multiple boggy scalp lesions for two months. Suspecting a bacterial etiology, the lesions were excised and sent for cultures. While bacterial cultures failed to grow, endothrix spores were readily detected in potassium hydroxide preparation and histopathology. Trichophyton tonsurans was identified by the phenotype method and later confirmed by matrix-assisted laser desorption ionization-time of flight (MALDI-TOF). Systemic antifungal therapy for 6 weeks along with local wound dressings resulted in complete recovery. At 2-year follow-up, focal alopecia was seen; however, no recurrence was noted.
\end{abstract}

\section{Introduction}

Kerion is an inflammatory fungal infection affecting the hair follicles of the scalp, characterized by boggy swelling, purulent discharge, alopecia, and cervical lymphadenopathy. Although more commonly noted in children, often it is an infrequent and, at times, difficult to treat, a condition of the scalp [1]. Of the different pathogens implicated in its cause, the dermatophytes of genera Trichophyton and Microsporum are considered the predominant ones [2].

We report a case of kerion, which was initially thought to be a pyogenic swelling of bacterial origin based upon its clinical characters and treated with surgical excision. The diagnosis was obtained on evaluation for fungal pathogenesis. We, therefore, recommend a strong index of clinical suspicion supported by relevant microbiological analysis to avoid its misdiagnosis.

\section{Case Illustration}

A previously healthy 9-year-old boy was evaluated for multiple painful scalp lesions for 2 months associated with focal alopecia. The lesions had started following a history of fall at the playground in the recent past. This was followed by the development of circular lesions that were itchy, to begin with. With failed attempts at treatment with oral antibiotics and topical antifungals, the lesions had increased in size evolving into boggy swellings with numerous pus points and black spots, as shown in Figure 1. The lesions were located over the parieto-occipital regions, the largest of which over the right parietal scalp measured $5 \times 5 \mathrm{~cm}$.

The hairs over the affected region were highly fragile, and the samples of hairs examined with $10 \%$ potassium hydroxide $(\mathrm{KOH})$ revealed endothrix spores along the hair shaft. 

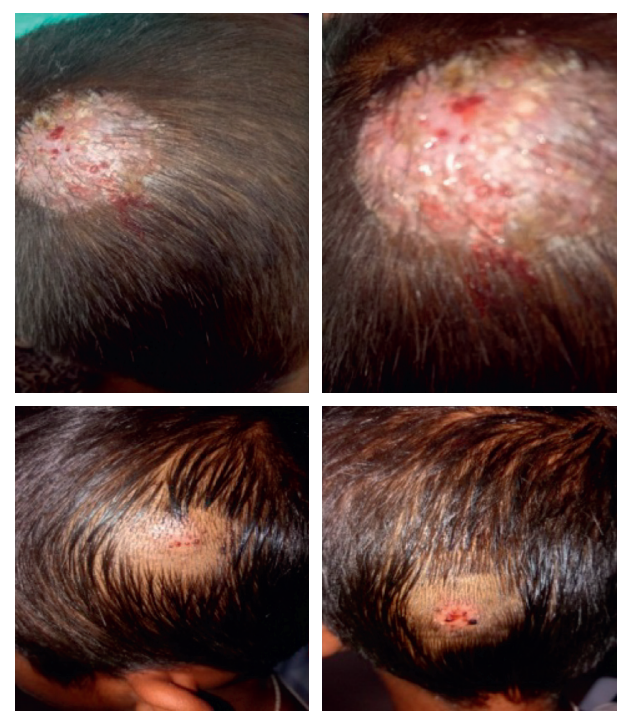

FIGURE 1: Boggy lesions over both the parietal scalp and occiput at presentation.

The excised lesions sent for bacterial culture were sterile. Fungal culture of the same on Sabouraud dextrose agar (SDA) grew small yellowish starry colonies on the $5^{\text {th }}$ day. On further incubation, the whitish colony with suede-like to powdery surface texture and radial furrows were seen. Also, the dermatophyte test medium (DTM) at $25^{\circ} \mathrm{C}$ identified the growth of dermatophyte, as shown in Figure 2. The pathogen was identified as Trichophyton tonsurans.

Lacto Phenol Cotton Blue (LPCB) mount revealed curved septate hyphae and other features characteristic of Trichophyton, as shown in Figure 3.

Tissue sent for histopathology revealed skin ulceration with marked neutrophilic infiltrates, lymphocytes, and plasma cells in the dermis, along with few aggregates of epitheloid histiocytes and giant cells.

Also, the typical presence of fungal spores inside the hair shaft was seen, as shown in Figure 4.

The isolate was confirmed by MALDI-TOF as Trichophyton tonsurans.

The patient received daily dressings for the next couple of days along with griseofulvin as systemic antifungal therapy for 6 weeks. A gradual and significant clinical improvement was noted, as shown in Figure 5.

He was followed up for two years during which no recurrence was noted. However, the lesions healed with focal alopecia.

\section{Discussion}

Dermatophytes are the most common cause of fungal infections worldwide, infecting millions of individuals annually. Of these, tinea capitis tends to affect mostly the prepubertal children in crowded communities in a background of low socioeconomic status [3].

Kerion Celsi, a typical form of tinea capitis, may develop as a T-cell-mediated hypersensitivity reaction [4] or as an abundant host immune response to fungal infection [5]. The

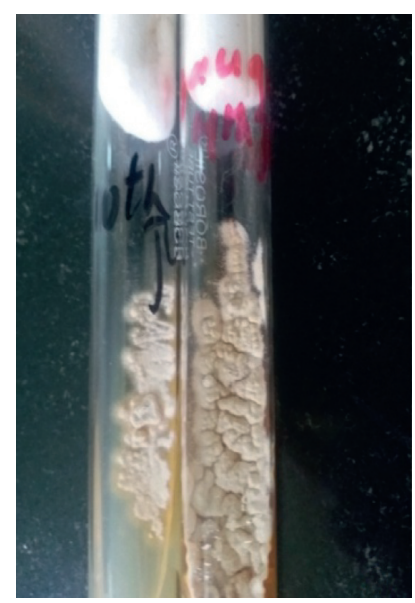

(a)

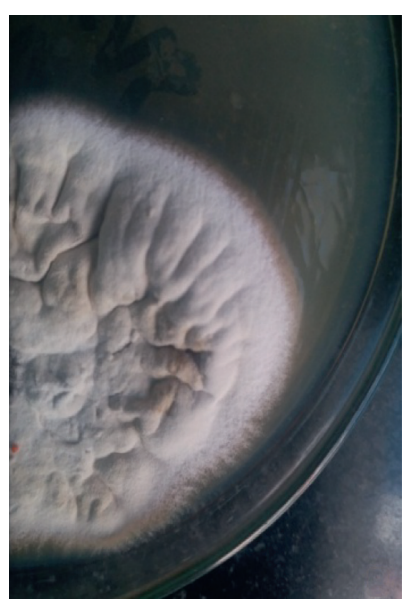

(b)
FIgure 2: (a) DTM; (b) SDA with dermatophyte growth and features of T. tonsurans.

classical kerion consists of a painful, boggy, inflammatory mass associated with alopecia that may be solitary or multiple or, sometimes, even suppurated and covered with a thick crust. Although tinea capitis can affect individuals of any age, with cases reported as early as six days of life and as late as 70 years of age, a vast majority of patients seem to be in the prepubescent group consisting of children between 3 and 7 years of age [6].

Most of the Trichophyton species are geophilic, of which T. tonsurans is said to easily survive outside the host and is reported to be the only anthropophilic fungus that has been isolated in soil samples [7]. The transmission of T. tonsurans may occur directly (by infected or asymptomatic human carriers) or indirectly (fomites). The possible source of the infection in our patient was probably the contact of the scalp with soil during the fall, leading to dermal implantation of the infective agent. Investigations were sent after the lesion was excised, suspecting a bacterial origin. Many scalp and hair disorders may mimic the clinical presentation of kerion such as seborrheic dermatitis, bacterial folliculitis, psoriasis, trichotillomania, alopecia areata, and discoid lupus erythematosus. As a result, kerion is often underrecognized or confused with other lesions, leading to misdiagnosis and a subsequently delayed or inappropriate treatment [8]. This has been identified by a few studies $[9,10]$. The burden of this disease has been estimated in 16 countries to date with a total of 21,073,423 cases, the majority of these being in subSaharan Africa and amongst school children. A recent review revealed a change in the last 1-2 decades and the spread of Trichophyton tonsurans as the dominant agent of tinea capitis, in the Americas, the UK, Europe, and Africa [11]. The burden of fungal infections in Nepal has long been underestimated with few diagnostic laboratories that are equipped with standard facilities to appropriately diagnose these conditions [12].

Once diagnosed, kerion can be treated with various systemic antifungal agents such as griseofulvin, terbinafine, itraconazole, or fluconazole. A 2008 meta-analysis had 


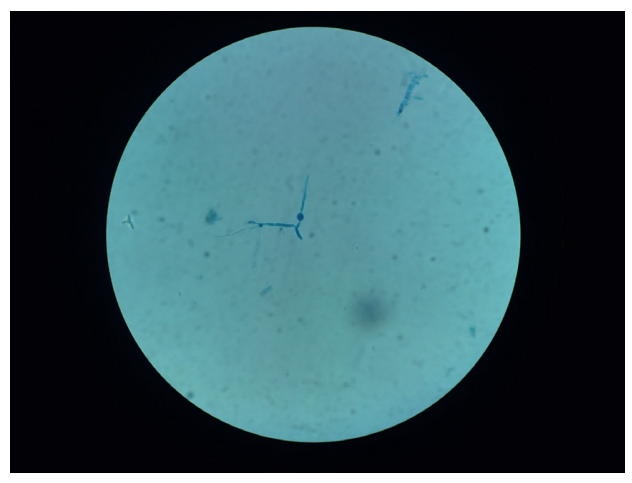

(a)

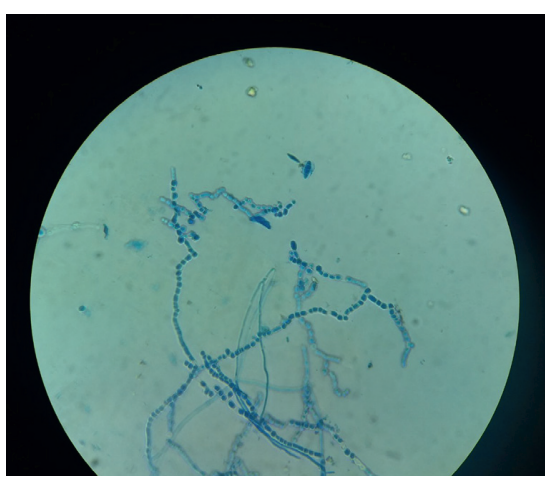

(b)

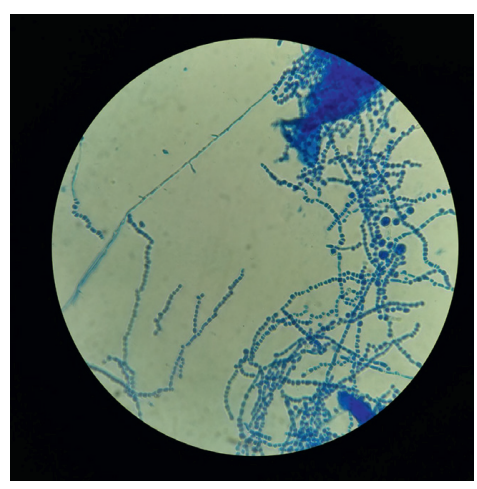

(c)

Figure 3: LPCB mount. (a) Curved septate hyphae as seen on the $5^{\text {th }}$ day. (b) (i) Abundant microconidia and few macroconidia. (ii) Intercalary and terminal chlamydospores. (c) Typical "bird on wire" pattern of microconidia.

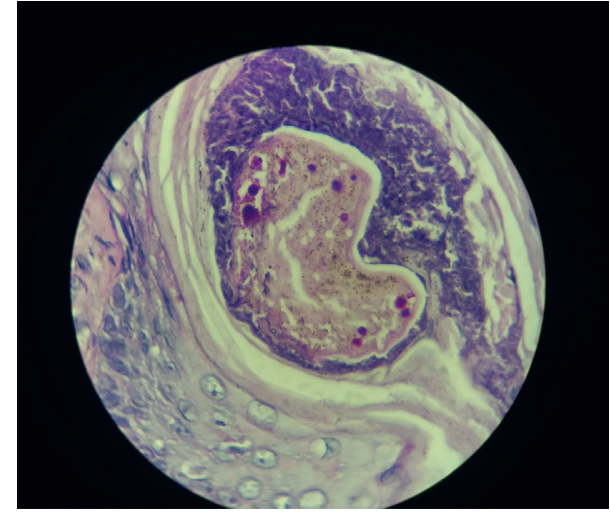

Figure 4: Histopathological features of the endothrix type of invasion.
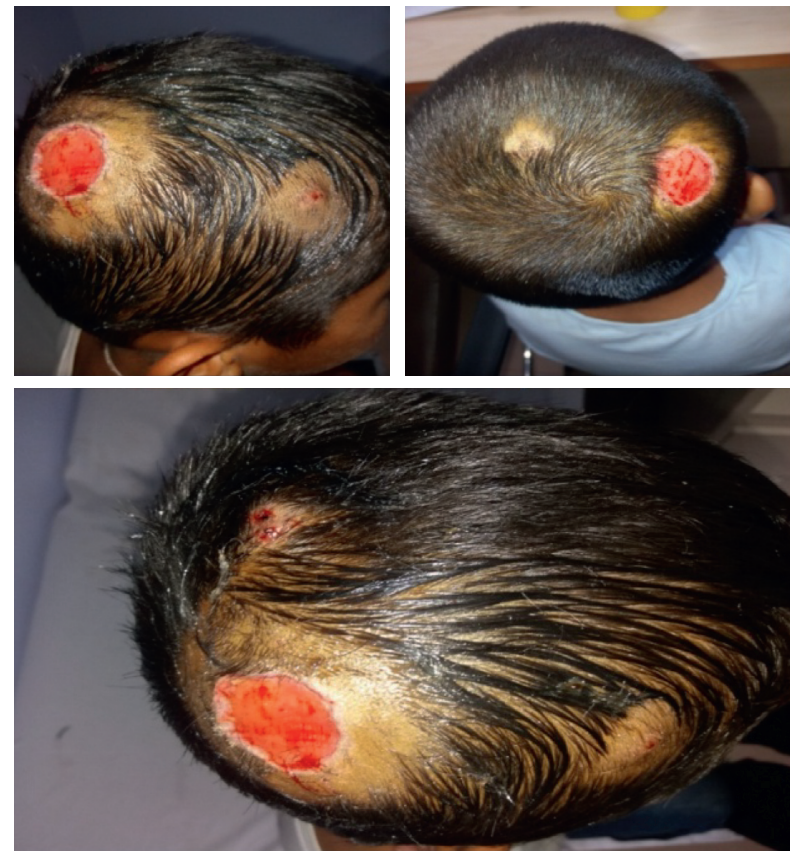

Figure 5: Follow-up at 1 week. found griseofulvin to be an effective therapy for tinea capitis [13]. Also, a Cochrane review had stated that the efficacy of these medications is similar in the treatment of tinea capitis due to the Trichophyton species [14]. Therefore, following the traditional treatment of choice, our patient was also administered griseofulvin with a satisfactory outcome at 2-year follow-up.

\section{Conclusions}

(1) High index of clinical suspicion is required to avoid misdiagnosis of kerion

(2) Clinical findings, $\mathrm{KOH}$ mount, and culture reports complement each other in the diagnosis

(3) Fungal culture is a necessary adjunct to direct microscopic examination for definitive identification of etiological agents

(4) The treatment can be more effective when antifungal therapy is based on the identification of fungal isolates

\section{Conflicts of Interest}

The authors declare that they have no conflicts of interest.

\section{Acknowledgments}

The authors acknowledge Dr. Arunaloke Chakrabrati, MD (President, International Society for Human Mycology), and his team of PGI Chandigarh for MALDI-TOF.

\section{References}

[1] L. V. Laer Tschudin and E. Laffitte, "Tinea capitis: no incision nor excision," Journal of Pediatric Surgery, vol. 42, no. 8, pp. E33-E36, 2007.

[2] R. J. Hay, “Tinea capitis: current status," Mycopathologia, vol. 182, no. 1-2, pp. 87-93, 2017.

[3] O. Ayanlowo, A. Akinkugbe, R. Oladele, and M. Balogun, "Prevalence of Tinea Capitis infection among primary school children in a rural setting in South-West Nigeria," Journal of Public Health in Africa, vol. 5, no. 1, 349 pages, 2014. 
[4] L. E. Proudfoot and R. Morris-Jones, "Kerion celsi," New England Journal of Medicine, vol. 366, no. 12, 1142 pages, 2012.

[5] J. T. Chussil, "Superficial fungal infection," in Dermatology For Advance Practice Clinicians, pp. 181-195, Wolters Kluwer, Philadelphia, PA, USA, 2015.

[6] M. L. Smith, "Tinea capitis," Pediatric Annals, vol. 25, no. 2, pp. 101-105, 1996.

[7] Z. B. S. Pontes and A. C. Oliveira, "Dermatophytes from urban soils in João pessoa, para'ıba, Brazil," Revista Argentina de Microbiolog' $1 a$, vol. 40, pp. 161-163, 2008.

[8] L. Trovato, S. Oliveri, M. Domina, I. Patamia, G. Scalia, and R. De Pasquale, "Molecular diagnosis of kerion celsi caused by Trichophyton tonsurans in a Italian child," Medical Mycology Case Reports, vol. 24, pp. 72-74, 2019.

[9] J. E. Feetham and N. Sargant, "Kerion celsi: a misdiagnosed scalp infection," Archives of Disease in Childhood, vol. 101, no. 5 , p. 503,2016

[10] V. Paudel, "Surgery of kerion, a nightmare for non- dermatologists," Case Reports in Dermatological Medicine, vol. 2020, Article ID 8825912, 3 pages, 2020.

[11] F. Bongomin, S. Gago, R. Oladele, and D. Denning, "Global and multi-national prevalence of fungal diseases-estimate precision," Journal of Fungi, vol. 3, no. 4, p. 57, 2017.

[12] N. Nayak, "Burden of fungal infections in Nepal," Nepal Journal of Epidemiology, vol. 6, no. 2, pp. 584-585, 2016.

[13] A. K. Gupta, E. A. Cooper, and J. E. Bowen, "Meta-analysis: griseofulvin efficacy in the treatment of tinea capitis," Journal of Drugs in Dermatology: JDD, vol. 7, no. 4, pp. 369-372, 2008.

[14] U. Gonzalez, T. Seaton, G. Bergus et al., "Systemic antifungal therapy for tinea capitis in children," Cochrane Database of Systematic Reviews, vol. 4, 2007. 